\title{
ANORMALIDADES DE ACROSSOMO E FERTILIDADE EM UM GARANHÃO: RELATO DE UM CASO
}

\author{
ACROSSOMAL ABNORMALITIES AND FERTILITY IN A STALLION: \\ A CASE REPORT
}

\section{Carlos Eurico Fernandes ${ }^{1}$ Cláudio Alves Pimentel ${ }^{2}$}

\section{RESUMO}

Descrevem-se os aspectos qualitativos do sêmen e a fertilidade de um garanhão da raça PSI (Puro Sangue Inglês), durante cinco períodos reprodutivos (1990 a 1994). Verificaramse altos índices de acrossomo anormais (13,6 a 39\%) nos ejaculados ( $N$ o = 15), na maioria do tipo "Knobbed Acrosome Defect" (área hipercromática ou espessamento excêntrico apical dos espermatozóides afetados. A porcentagem de prenhez $(P)$ variou entre 25 e $90 \%$ e a porcentagem de prenhez por ciclo $(P /$ $C)$ entre 20 e 59\%. Através de análise de regressão, $P / C$ relacionou-se significativamente $(p<0,05)$ com os indices de acrossomos anormais $\left(R^{2}=0,87\right)$. As demais características seminais avaliadas, não se relacionaram aos índices de fertilidade. Conclui-se que o defeito de acrossomo, neste caso, podem ser atribuídas à espermiogênese imperfeita. Tal anormalidade afeta significativamente a fertilidade do garanhão.

Palavras-chave: garanhão, acrossomo, sêmen, fertilidade.

\section{SUMMARY}

It is described certain qualitative seminal characteristics and the fertility of a Thoroughbred stallion, during five consecutive breeding seasons (1990 a 1994). High percentages (13.6 a 39\%) of abnormal acrossomes were observed in the ejaculates $\left(N^{o}=15\right)$, being the majority of the "Knobbed Acrosome Defect" type (dark staining area or eccentric thickening at the apex of affected spermatozoa). The pregnancy rate $(P)$ varied between 25 e $90 \%$ and the per cycle pregnancy $(P / C)$ between 20 e 59\%. Regression analysis showed that P/C was significantly $(p<0.05)$ related to the percentage of abnormal acrossomes $\left(R^{2}=0.87\right)$. Other seminal characteristics considered in this report were not associated $(P>0.05)$ to fertility indexes. The conclusion is that the acrossomal defect, in the present case, could be attributed to arrested spermiogenesis. Such abnormality significantly affected stallion fertility.

Key words: stallion, acrossome, semen, fertility.

\section{INTRODUÇÃO}

A avaliação da integridade do acrossomo é um importante aspecto da estimativa da fertilidade potencial do macho, já que possui enzimas que participam ativamente no processo de fertilização. A ocorrência de uma certa proporção de acrossomos anormais no ejaculado indica que uma considerável parcela da população espermática, além daqueles que, através do microscópio ótico apresentam o defeito, devem ter alterações quantitativas e/ou qualitativas de suas enzimas, com uma correspondente redução nos índices de fertilidade. Dentre os diferentes tipos de anormalidades acrossômicas que ocorrem em diversas espécies animais, comprometendo a fertilidade (HANCOCK \& TREVAN, 1957; SAACKE $\boldsymbol{e t}$ al., 1968; HURTGEN \& JOHNSON, 1982), está o tipo

${ }^{1}$ Médico Veterinário autônomo, MMV

${ }^{2}$ Médico Veterinário, PhD., Professor Titular do Departamento de Patologia Animal, Faculdade de Veterinária, UFPel, 96010-900 Pelotas, RS. Autor para corrrespondência. 
chamado "Knobben Acrossome Defect" (BLOM \& BIRCH-ANDERSEN, 1962). Trata-se de uma área hipercromática ou de espessamento excêntrico na extremidade do espermatozóide afetado; vesículas ou inclusões membranosas ficam envoltas no interior da matriz acrossomática (BARTH \& OKO, 1989). Geralmente esse defeito ocorre associado a casos de degeneração testicular e acompanha a elevação de outras anormalidades morfológicas do sêmen (FRIEDMAN et al., 1991). Entretanto, há casos em que ocorrem como o principal ou mesmo único defeito significativo no ejaculado e com uma freqüência constante, tendo sido diagnosticado no touro, como Espermiogênese Imperfeita (GARCIA, 1971).

No garanhão, GRONDAHL $\boldsymbol{e t} \boldsymbol{a l} .$, (1992) verificaram que as alterações mais comuns estão associadas a formação de vesículas nas membranas acrossomáticas ("pouch formation"), com freqüência entre 16 e $24 \%$. Entretanto, de modo geral, os percentuais de espermatozóides com anormalidades no acrossomo encontrados em garanhões de alta fertilidade são baixos (DOTT, 1975; BIELANSKI, 1982) e usualmente não ultrapassam a $5 \%$ (PIMENTEL, 1989).

Em função disto, poucos casos tem sido descritos ressaltando a eficiência reprodutiva de garanhões com índices elevados de acrossomos anormais no ejaculado. HURTGEN \& JOHNSON (1982) estudaram garanhões que apresentavam entre 27 e $74 \%$ de espermatozóides com "knobbed defect" e HELD et al. (1991) descreveram a presença de vacúolos na região acrossomática em um garanhão da raça Árabe. No entanto, em nenhum destes estudos foi possível estimar com precisão o efeito destas anormalidades na fertilidade obtida por cada garanhão. De modo geral são explorados apenas os aspectos ligados à morfologia (DOTT, 1975; DOWSETT et al., 1984; GRONDAHL et al., 1992; VEERMACHANENI RAO $\boldsymbol{e}$ t al., 1993)

O propósito deste estudo é avaliar a fertilidade de um garanhão com altos percentuais de espermatozóides com anormalidades de acrossomo, bem como estabelecer a relação entre esta anormalidade e os índices reprodutivos, baseados na porcentagem de prenhez $(\mathrm{P})$ e porcentagem de prenhez por ciclo $(\mathrm{P} / \mathrm{C})$.

\section{RELATO DE CASO}

Durante as temporadas reprodutivas (agosto à dezembro) de 1990 a 1994, acompanhou-se a fertilidade e as características seminais de um garanhão da raça PSI, nascido em 1982 e de propriedade particular. Durante este período o animal apresentou boas condições clínicas, sem evidências de doença infecto-contagiosa. A cada coleta de sêmen era feito um exame clínico dos órgãos reprodutivos externos e estes sempre se apresentaram dentro de parâmetros previamente descritos por KENNEY et al. (1983) e MERKT \& KLUG (1989). Os testículos mostravam-se simétricos, com boa mobilidade na bolsa escrotal e de consistência fibro-elástico. O epidídimo era do tamanho aproximado de uma noz e de consistência firme.

O sêmen era coletado através de vagina artificial, modelo Colorado (PICKETT et al. 1970). Em $1990 \mathrm{e}$ 1991, o ejaculado foi obtido próximo ao final da temporada reprodutiva, enquanto nos demais (1992 a 1994) foram obtidos durante o decorrer dos respectivos períodos. Todas as coletas foram feitas sem que o garanhão estivesse em repouso sexual por mais de quatro dias.

Após a coleta, a fração rica em esperma era separada da porção gelatinosa através de um filtro adaptado no próprio copo coletor. Em seguida, o volume da fração sem gel era medido e a porção gelatinosa, desprezada. A avaliação da motilidade (porcentagem de células móveis por campo) e da concentração espermática foi conduzida segundo KENNEY et al.(1983). A determinação da morfologia espermática foi feita em microscopia de contraste de fase, a partir de amostras conservadas em solução de formol salina tamponada (HANCOCK, 1957). Eram examinados 200 espermatozóides em cada lâmina, em aumento de 1000 vezes, obtendo-se os percentuais de células normais e das seguintes anormalidades: cabeça, peça intermediária, cauda, gota proximal, gota distal, cabeça isolada normal e acrossomo (KENNEY et al. 1983). O total de espermatozóides viáveis, em cada ejaculado, foi estimado com base no total ejaculado, percentual de móveis e de normais (ajustado pelos índices de anormalidades de cauda e cabeça isolada normal), segundo KENNEY (1989).

A fertilidade em cada ano, foi estimada através da $\mathrm{P}$ e $\mathrm{P} / \mathrm{C}$, obtidos no final de cada período reprodutivo. Éguas com história de subfertilidade (mais de dois anos sem conceber) ou que apresentavam problemas reprodutivos (alterações inflamatórias, disturbios endócrinos, distocia, retenção de placenta ou que tenham sido submetidas a qualquer tipo de tratamento por problemas genitais) foram descartadas. O diagnóstico de gestação era feito através da ultrasonografia, a partir do $14^{\circ}$ dia pós-ovulação e repetido em intervalos de 7 a 10 dias até aproximadamente 50 dias de gestação. 
O modelo matemático que estabeleceu a relação entre as características seminais (variáveis independentes) e os índices de fertilidade (variáveis dependentes), obtidos a cada temporada, foi estimado através da análise da regressão múltipla "stepwise" (DRAPER ; SMITH, 1980).

\section{RESULTADOS E DISCUSSÃO}

Na Tabela 1 estão descritos os valores das características seminais, assim como a média geral e respectivo desvio, obtidos durante as cinco temporadas reprodutivas. As formas anormais de maior freqüência foram os espermatozóides tipo "Knobbed" (condensação na região acrossomática), embora outras anormalidades como "fold acrosome" (acrossomo dobrado) também foram encontradas (Figura 1).

Nas temporadas de 1991 e 1992 verificou-se uma elevação nos percentuais de acrossomo anormais (32,0 e 39,0, respectivamente), coincidindo com o decréscimo de P (47 e 25\%) e P/C (36 e 20\%) descritos na Tabela 2. Nos anos seguintes, 1993 e 1994, a fertilidade aumentou progressivamente, enquanto as anormalidades de acrossomo diminuíram.

O percentual de acrossomos anormais e a fertilidade variaram entre as cinco temporadas estudadas, porém, características como a motilidade, concentração espermática e demais formas anormais, mantiveram-se estáveis durante este período, permanecendo dentro dos limites

Tabela 1 - Valores das características seminais durante o periodo observado.

Temporada

\begin{tabular}{lccccccc}
\hline \multicolumn{1}{c}{ Características } & 1990 & 1991 & 1992 & 1993 & 1994 & Média & DP \\
& $(\mathrm{n}=1)$ & $(\mathrm{n}=1)$ & $(\mathrm{n}=9)$ & $(\mathrm{n}=2)$ & $(\mathrm{n}=2)$ & & \\
Volume (ml) & 50,0 & 60,0 & 84,2 & 52,5 & 61,0 & 71,3 & 28,5 \\
Motilidade $(\%)$ & 50 & 40 & 48 & 45 & 45 & 47 & 11,8 \\
Concentração(10\%/ml) & 327 & 48,5 & 130 & 194 & 149 & 151,7 & 88,9 \\
Total Ejaculado(x $\left.10^{5}\right)$ & 16,3 & 2,9 & 10,9 & 10,2 & 9,0 & 9,8 & 4,9 \\
Total viáveis $\left(\mathrm{x} 10^{5}\right)$ & 7,2 & 0,65 & 2,5 & 2,7 & 2,0 & 2,8 & 1,9 \\
Morfologia (\%) & & & & & & & \\
Normais & 65,5 & 49,5 & 43,8 & 54,5 & 54,0 & 46,8 & 10,1 \\
Defeitos de Cabeça & 4,0 & 7,5 & 8,6 & 5,5 & 11,0 & 8,3 & 5,1 \\
Defeitos de Peça & & & & & & & \\
$\quad$ Intermediária & 1,5 & 4,0 & 0,6 & 6,2 & 2,5 & 1,4 & 1,9 \\
Defeitos de Cauda & 6,0 & 3,0 & 1,1 & 4,0 & 3,0 & 1,8 & 1,7 \\
Defeitos de Acrossomos & 15,0 & 32,0 & 39,0 & 22,7 & 22,5 & 34,7 & 13,6 \\
Cabeça Isolada Normal & 1,0 & 1,5 & 2,8 & 1,7 & 2,0 & 2,5 & 2,3 \\
Gota Proximal & 0,5 & 0,5 & 0,4 & 0,2 & 0 & 0,4 & 0,5 \\
Gota Distal & 2,5 & 1,0 & 0,6 & 3,7 & 5,0 & 1,3 & 2,0 \\
& & & & & & & \\
\hline
\end{tabular}

$\mathrm{n}=$ número de ejaculados avaliados, $\mathrm{m}=$ média, $\mathrm{d} p=$ desvio padrão da média

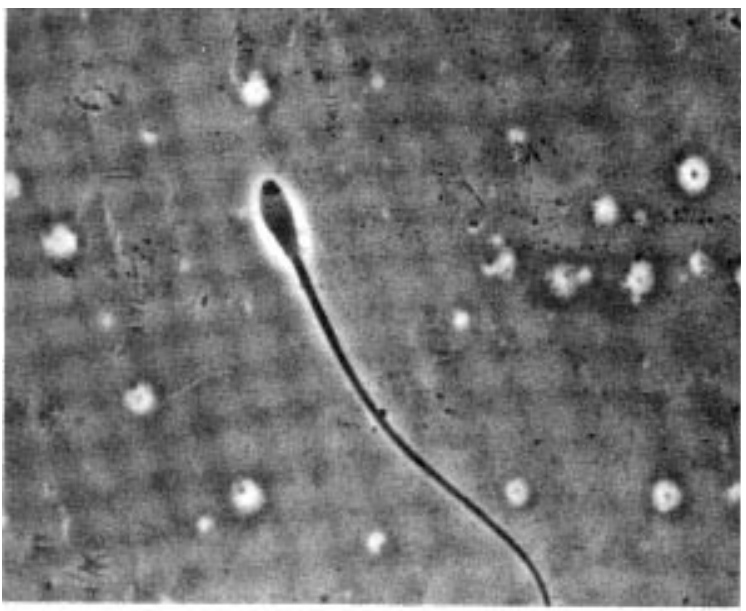

Figura 1 - Defeito de Acrossomo típico do espermograma do garanhão.

previamente reportados por NISHIKAWA (1959), DOWSETT; PATTIE (1982), PICKETT et al. (1989) JASKO et al. (1990). Isto sugere que a alta prevalência de acrossomos com "knobbed defect", encontrada em todos os ejaculados, não esteve diretamente ligada a um processo de degeneração testicular. Em outros estudos (HURTGEN \& JOHNSON, 1982 e VEERANACHANENI RAO $\boldsymbol{e} t$ al. 1993), a diminuição da motilidade e concentração espermática, juntamente com altos percentuais de anormalidades de cabeça, peça intermediária (massa microtubular), gota proximal, além da presença de fragmentos de espermatócitos e células de Sertoli degeneradas, caracterizaram um processo avançado de degeneração do epitélio germinativo. Também em outras espécies, como bovinos, ovinos e suínos, a alta prevalência de acrossomo anormais tem sido encontrada em associação com outras anormalidades no quadro seminal e conseqüente subfertilidade (HANCOCK, 1949; OTT et al. 1982; BARTH; OKO, 1989).

Embora as alterações de acrossomo tenham origem durante a espermiogênese (FAWCETT, 1958; JOHNSON, 1991), a causa exata ainda não está totalmente

esclarecida.

Ciência Rural, v. 27, n. 2, 1997. 


\begin{tabular}{|c|c|c|c|c|c|}
\hline \multirow[b]{2}{*}{ Temporada } & \multirow[b]{2}{*}{$\begin{array}{l}\text { Éguas } \\
\text { servidas }\end{array}$} & \multirow[b]{2}{*}{ prenhes } & \multirow[b]{2}{*}{ ciclos } & \multicolumn{2}{|c|}{ Índices $(\%)$} \\
\hline & & & & $P$ & $\mathrm{P} / \mathrm{C}$ \\
\hline 1990 & 24 & 19 & 32 & 79 & 59 \\
\hline 1991 & 45 & 21 & 59 & 47 & 36 \\
\hline 1992 & 59 & 15 & 85 & 25 & 20 \\
\hline 1993 & 35 & 21 & 60 & 60 & 35 \\
\hline 1994 & 32 & 29 & 51 & 90 & 57 \\
\hline Total & 195 & 105 & 287 & 54 & 37 \\
\hline
\end{tabular}

Aspectos associados ao potencial reprodutivo das éguas, técnicas de cobertura, manejo geral a que os animais são submetidos, entre outros, também tem sido descritos como importantes aspectos a serem considerados na variação da fertilidade do garanhão (VOSS, 1993). Porém, é possível concluir que a espermiogênese imperfeita, neste caso representada pela elevação do percentual de acrossomos com condensação na região apical ("knobbed acrosome defect"), pode representar uma significativa parcela da variação na eficiência reprodutiva de um programa.

\section{REFERÊNCIAS BIBLIOGRÁFICAS}

BARTH, A.D., OKO, R.J. Defects of the sperm head. In: Barth, A.D., Oko, R.J. Abnormal morphology of bovine spermatozoa. Iowa State University press/Ames, $1^{\mathrm{a}}$ ed, USA, p. $130-192,1989$.

KENNEY \& MOYER (1973) descreveram um caso de espermiogênese imperfeita onde eram observados além de acrossomos anormais, espermatozóides com hipoplasia de peça intermediária ("tail stump"), possivelmente de natureza congênita ou hereditária. Em touros, VIERULA et al. (1987) descreveram transtornos de espermiogênese ligados a um gene autossômico recessivo. Segundo HURTGEN \& JOHNSON (1982) as anormalidade de acrossomos podem ser induzidas pela maturação incompleta de uma determinada população de espermatozóides.

A fertilidade com base no números de ciclos $(\mathrm{P} / \mathrm{C})$ tem sido usada no estudo da fertilidade do garanhão, já que representa mais acuradamente a eficiência reprodutiva em um determinado período (KENNEY et al. 1983; VOSS, 1993). Ao contrário, P reflete apenas a proporção de éguas que concebem. Mesmo assim, ambas variáveis indicaram uma baixa fertilidade, já que aproximadamente $50 \%$ da éguas servidas conceberam $(\mathrm{P}=54 \%)$, levando ao redor de 3 ciclos por concepção $(\mathrm{P} / \mathrm{C}=37 \%)$. Através da análise da regressão entre as características seminais e índices de fertilidade, verificou-se que apenas P/C (Y) relacionou-se significativamente $(\mathrm{p}<0,05)$ com o percentual de acrossomo anormais $(\mathrm{X})$, expressada pela equação $\mathrm{Y}=83,58-1,43 \mathrm{X}\left(\mathrm{R}^{2}=0,87\right)$. Embora várias características seminais tenham sido associadas à fertilidade (JASKO et al. 1990; 1992), os resultados aqui obtidos permitiram estimar com uma boa margem de segurança que o aumento de uma unidade no percentual de acrossomos anormais induziu a um decréscimo de $1,43 \%$ na fertilidade baseada na $\mathrm{P} / \mathrm{C}$.
BIELANSKI, W., DUDEK, E., BITTMAR, A. et al. Some characteristics of common abnormal forms of spermatozoa in high fertile stallions. J Reprod Fert Sup, v. 32, p. 21-26, 1982 .

BLOM, E, BIRCH-ANDERSEN, A. Ultrastructure of the sterilizing knobbed sperm defect in the bull. Nature, v. 194, p. 989-990, 1962.

DOTT, H.M. Morphology of stallion spermatozoa J Reprod Fertil, Suppl., v. 23, p. 41-46, 1975.

DOWSETT, K.F., PATTIE, W.A. Characteristics and fertility of stallion semen. J Reprod Fertil, Suppl. v. 32, p. 1-8. 1982.

DOWSETT, K.F., OSBORNE, H.G., PATTIE, W.A. Morphological characteristics of stallions spermatozoa. Theriogenology. v. 22, p. 463-472, 1984.

DRAPER, N.R., SMITH, H. Applied Regression Analysis. John Wiley, Sons, USA, 2a ed., 709 p. 1980.

FAWCETT, D.W. The structure of the mammalian spermatozoa. Int Rev Cytol, v. 7, p. 195-235, 1958.

FRIEDMAN R., SCOTT M., HEATH S.E., et al. 1991. The effects of increased testicular temperature on spermatogenesis in the stallion. J Reprod Fertil, (Suppl. 44):127-134.

GARCIA, O.S. Aspectos físicos e morfológicos do sêmen de touros. Tese Mestrado. Belo Horizonte, MG. 61 p. 1971.

GRONDAHL, C., HYTTEL, P., GREVE, T. Acrossome status in fresh and frozen thawed stallion spermatozoa evaluated by scanning electron microscopy. 12th International Congress on Animal Reproduction. v. 4, p. 1870-1872, 1992.

HANCOCK, J.L. Evidence of an inherited seminal character associated with infértil HANCOCK, J.L. Morphology of boar spermatozoa. J Microsc Soc, v. 76, p. 84-97, 1957. 
HANCOCK, J.L., TREVAN, D.J. The acrossome and post-nuclear cap of bull spermatozoa. J R Microsc Soc, v. 76, p. 77, 1957.

HELD, J.P., PRATER, P., STETTLER, M. Spermatozoal head defects as a cause of infertility in a stallion. J Am Vet Med Assoc v. 199, p. 1760-1761, 1991.

HURTGEN, J.P., JOHNSON, L.A. Fertility of stallions with abnormalities of the sperm acrossome. J Reprod Fertility, Sup. v. 32, p. 15-20, 1982

JASKO, D.J., LEIN, D.H., FOOT, R.H. Determination of the relationship between sperm morphologic classifications and fertility en stallions: 66 cases (1987-1988). J Am Vet Med Assoc, v. 197, p. 389-394, 1990.

JASKO, D.J., LITTLE, T.V., LEIN, D.H., et al. Comparison of spermatozoa movement and semen characteristics with fertility in stallions. 64 cases (1987-1988). J Am Vet Med Assoc, v. .200 , p. 979-985, 1992.

JOHNSON, L Spermatogenesis. In: CUPPS, P.T. Reproduction in domestic animal. 4a ed. Academic Press, San Diego, CA. p. $173-219,1991$

KENNEY, R.M., MOYER, W. Clinic-pathologic Conference.J Am Vet Med Assoc, v. 162, p. 142-147, 1973.

KENNEY, R.M. VIII Congresso Brasileiro de Reprodução Animal. Curso de reprodução em eqüinos Belo Horizonte, 1989.

KENNEY, R.M., HURTGEN, J.P., PERSON, R., et al. Society for Theriogenology: manual for clinical fertility evaluation of the stallion Hastings, NB, 1983, $100 \mathrm{p}$.

MERKT, H., KLUG, E. Gesundheitliche und geschlechtliche Mindestanforderungen an Zuchthengste. Dtsch Tierarztl Wschr v. 96, p. 433-472, 1989.
NISHIKAWA, Y. Studies on reproduction in horses singularity and artificial control in reproduction phenomena Koei Kvoto, Tokyi, Japan , 1959, 340 p.

OTT, R.S., HEATH, E.H., BANE, A. Abnormal spermatozoa, testicular degeneration and varicocele in a ram Amer $\mathbf{J}$ Vet Res,v. 42, p. 241-245, 1982.

PICKETT, B.W., AMANN, R.P., McKINNON, A.O., et al. Management of the stallion for maximum reproductive efficiency, II Anim Reprod Lab Fort Collins, CO, 1989, 126 p.

PICKETT, B.W., FAULKNER, L.C., SUTHERLAND, T.M. Effect of month and stallion on seminal characteristics and sexual behavior J Anim Sci , v. 31, p. 713-728, 1970.

PIMENTEL, C.A. Aspectos da patologia espermática e a fertilidade no garanhão Congresso Brasileiro de Reprodução Animal, v. 8 , p. $127-132,1989$

SAACKE, R.G., AMANN, R.P., MARSHALL, C.E. Acrossomal cap abnormalities of sperm from subfertile bulls J Anim Sci, v. 27, p. 1391-1399, 1968

VEERAMACHANENI RAO, D.N., MOELLER, C.L., SHINER, K. A., et al. On processing and evaluation of equine seminal samples for cytopathology and fertility assessment: the utility of electron microscopy J Equine Vet Science, v. 13, p. 207-215, 1993

VIERULA, M., ALANKO, M., ANDERSON, M., et al. Tail stump sperm defect in Ayrshire bulls: morphogenesis of the defect Andrologia, v. 19, p. 207-216, 1987.

VOSS, J.L. Breeding efficiency In: McKINNOW, A.O., VOSS, J. L. Equine Reproduction, Philadelphia, Lea, Febiger, 1993, 1109 p. 